\title{
CARBON ISOTOPIC CHEMISTRY
}

\author{
W. D. LANGER \\ MS $169-506$ \\ Jet Propulsion Laboratory \\ California Institute of Technology \\ Pasadena, CA 91109 USA
}

\begin{abstract}
Isotopic molecular abundances are used to interpret Galactic chemical evolution and the properties of interstellar clouds. The isotopic chemistry of carbon plays an important role in the interpretation of these measurements. This paper reviews the recent measurements of the carbon twelve to thirteen ratio across the Galaxy and the isotopic chemistry.
\end{abstract}

\section{Introduction}

For twenty years isotopic abundances of molecules in interstellar clouds (ISC) have been measured by radio and optical techniques. These observations are important to: (1) determine Galactic chemical evolution (nucleosynthesis, star formation rate, injection and mixing of gas in the interstellar medium); (2) interpret physical conditions in ISCs; (3) test models of interstellar chemistry; and, (4) study the chemical evolution of the solar system. Carbon isotopic species are especially important because carbon molecules are pervasive and important probes of ISCs, the chemistry is reasonably well understood, and twelve and thirteen carbon trace primary and secondary nucleosynthesis, respectively. Here I review carbon isotopic ratio measurements and discuss the basic elements of the carbon isotopic chemistry

\section{Observations of Carbon Isotopic Ratios}

Across the Galaxy carbon isotopic ratios have been measured in the radio, mainly with $\mathrm{CO}$ and $\mathrm{H}_{2} \mathrm{CO}$, and locally $(<1 \mathrm{kpc})$ also measured in the optical with $\mathrm{CH}^{+}$and $\mathrm{CN}$. $\mathrm{CO}$ is pervasive and easily observed in emission, however the most abundant isotopes have large optical depths and good determinations of a ratio depend on detecting the very weak ${ }^{13} \mathrm{C}^{18} \mathrm{O}$ and correcting for ${ }^{12} \mathrm{C}^{18} \mathrm{O}$ opacity. Formaldehyde is observed in absorption towards strong continuum sources in its ${ }^{12} \mathrm{C}$ and ${ }^{13} \mathrm{C}$ isotopes. To use this species one must deal with the weakness of $\mathrm{H}_{2}{ }^{13} \mathrm{CO}$, photon trapping corrections for $\mathrm{H}_{2}{ }^{12} \mathrm{CO}$, and the uncertain effects of clumpiness on the trapping correction. In addition chemical processes can enhance isotopic ratios in these molecules. The optical absorption studies generally 
have poor velocity resolution, though some high velocity resolution measurements have been made (limited to very bright nearby sources). The determination of ratios is complicated by baseline subtraction and line fitting (cf. Vladilo and Centurion 1991). Isotopic enhancement is probably not important for $\mathrm{CH}^{+}$formation towards these sources, but may be a factor for $\mathrm{CN}$ and $\mathrm{CO}$.

To address the issue of the carbon isotopic ratio across the Galaxy, Langer and Penzias (1990; hereafter LP90) recently presented a study of nine interstellar clouds observed in the rare and doubly rare isotopes of carbon monoxide, ${ }^{12} \mathrm{C}^{18} \mathrm{O}$ and ${ }^{13} \mathrm{C}^{18} \mathrm{O}$. The use of low abundance species minimizes radiative transfer effects, and observations towards dense shielded cores reduces complications from isotopic chemical and photodissociation enhancements. They found a systematic gradient in the ${ }^{12} \mathrm{C} /{ }^{13} \mathrm{C}$ ratio across the Galaxy from $\approx 30$ in the inner part at $5 \mathrm{kpc}$ to $\approx 70$ at $12 \mathrm{kpc}$. This trend is similar to that derived from formaldehyde observations (Henkel, Wilson, and Beiging 1982), however the formaldehyde ratios are generally higher across the disk, and show more scatter than the $\mathrm{CO}$ measurements. Both the $\mathrm{CO}$ and formaldehyde indicate a Galactic Center value about 24. Figure 1 displays the isotope ratio from formaldehyde and carbon monoxide across the Galaxy as a function of distance, D, from the Galactic center) along with the corresponding linear fits $\left({ }^{12} \mathrm{C} /{ }^{13} \mathrm{C}\right.$ proportional to $5.6 \mathrm{D}(\mathrm{kpc})$ for $\mathrm{H}_{2} \mathrm{CO}$ and $5.9 \mathrm{D}(\mathrm{kpc})$ for $\mathrm{CO}$

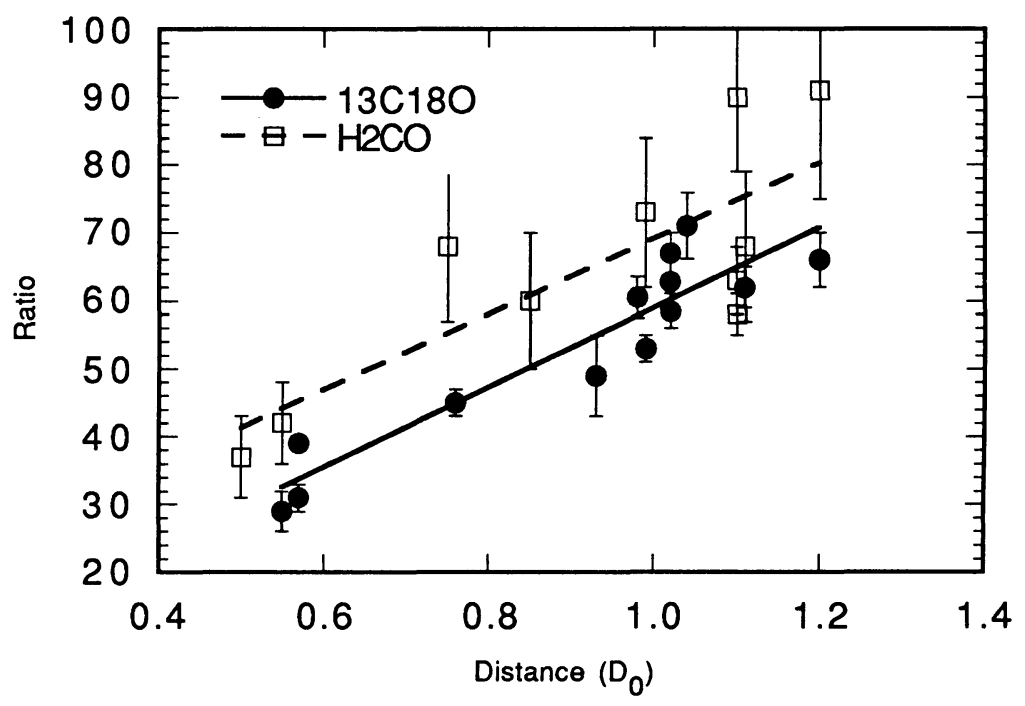

Fig. 1. Carbon 12 to 13 isotope ratio across the Galaxy using $\mathrm{H}_{2} \mathrm{CO}$ (Henkel et al. 1982) and CO (LP90 and LP91) data, where distance is with respect to Solar distance $\mathrm{D}_{0}$.

In the local solar neighborhood LP90 derived an average value ${ }^{12} \mathrm{C} /{ }^{13} \mathrm{C}=57 \pm 3$ from the Galactic gradient, although individual sources ranged from 49 to 67 (excluding 79 in the Orion A K-L region because the strong nearby HII region produces photodissociation enhancement effects). Because there are a wide variety of techniques and species available for measuring the ratio in the solar neighborhood accurate determinations in this region are 
particularly important for fixing the local value, thereby establishing absolute values across the Galaxy.

To improve the value for the carbon isotope ratio in the local solar neighborhood Langer and Penzias (1991; hereafter LP91) extended their ${ }^{13} \mathrm{C}^{18} \mathrm{O}$ and ${ }^{12} \mathrm{C}^{18} \mathrm{O}$ study to three sources not known to be associated with HII regions or strong UV radiation fields: B5, B335, and L134N (also known as L183). In addition they observed another position in Orion far from the HII region near K-L. A sample spectrum from B5 is shown in Figure 2. In the four sources the ratio ranges from 58 to 71 with a weighted average of $61 \pm 2$ (plotted in Figure 1), slightly larger than the value of 57 suggested in the earlier work on the Galactic gradient. The local value of $61 \pm 2$ derived from $\mathrm{CO}$ is considerably smaller than the formaldehyde result of $80 \pm 7$, as well as the terrestrial value of 89 .

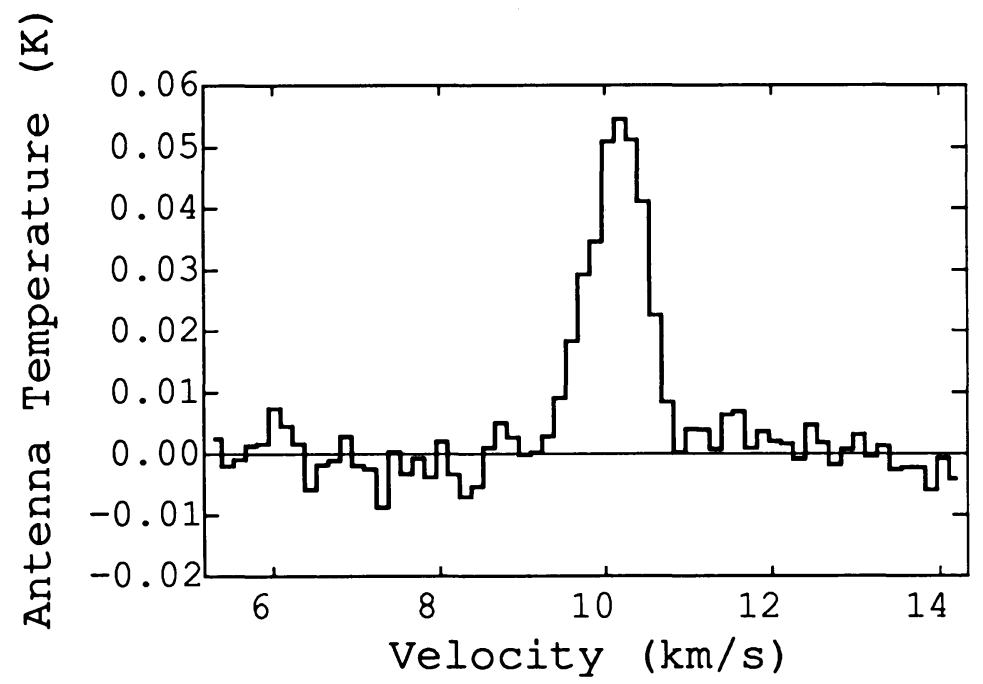

Fig. 2 Spectrum of ${ }^{13} \mathrm{C}^{18} \mathrm{O}$ in the source $\mathrm{B} 5$.

The optical measurements of the isotope ratio have improved in recent years but also show considerable differences among various observations of $\mathrm{CH}^{+}$and $\mathrm{CN}$. Kaiser, Hawkins, and Wright (1991) summarize recent optical observations in the local solar neighborhood (see their Table 1). The isotope value derived from $\mathrm{CH}^{+}$is not without controversy. Stahl et al. (1989), Crane et al. (1991), and Stahl and Wilson (1991) find a value about $67 \pm 2$, in agreement with earlier (but less accurate) measurements. While the

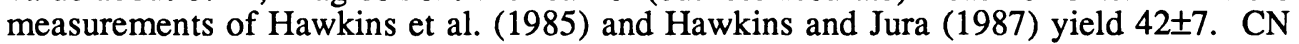
absorption line observations of Crane and Hegyi (1988) yield 47 \pm 5 . Furthermore, Hawkins and Jura (1987) derive a local value of $43 \pm 4$ from averaging several $\mathrm{CH}^{+}$sources within a kpc. Recently Vladilo and Centurion (1991) suggested that the data analysis approach of Hawkins and co-workers could overestimate the equivalent width for ${ }^{13} \mathrm{CH}^{+}$ by a factor of two, perhaps explaining the lower values. It also differs somewhat from the best values derived from the $\mathrm{CH}^{+}$absorption measurements of the nearby cloud Zeta Oph, $71 \pm 3$ (Stahl and Wilson 1991) to $67 \pm 2$ (Crane et al. 1991). 
These radio and optical results raise the following important questions. 1) Why are there differences between the $\mathrm{CO}$ and $\mathrm{H}_{2} \mathrm{CO}$ ? (2) Why do $\mathrm{CN}$ and $\mathrm{CH}^{+}$isotope ratios differ? The answer may lie in the details of the isotopic chemistry.

\section{Carbon Isotopic Chemistry}

Isotopic reactions are important both in shielded regions where cosmic ray ionization drives the ion molecule chemistry of carbon and in translucent or PDR regions where UV radiation is important. In dense shielded regions cosmic rays produce $\mathrm{H}_{3}{ }^{+}$ions which through their interactions with $\mathrm{O}$ and $\mathrm{C}$ initiate molecule production. Most of the carbon ends up as $\mathrm{CO}$ (assuming there is more gaseous oxygen available than carbon), with small amounts found in other molecules such as formaldehyde, CS, and CN . A wide variety of neutral-neutral, electron recombination, and ion-molecule reactions are involved in the chemistry once the initiating steps are taken (cf. Prasad and Huntress 1980; Langer et al. 1984; Langer and Graedel 1989). Helium ions destroy the stablest molecules and in the case of $\mathrm{CO}$ produce carbon ions. The balance between $\mathrm{H}_{3}{ }^{+}$converting carbon and oxygen atoms into molecules and $\mathrm{He}^{+}$destroying the most stable molecules $\mathrm{CO}, \mathrm{O}_{2}$, and $\mathrm{H}_{2} \mathrm{O}$, for example, determines the relative abundance of atomic to molecular form. Isotopic exchange reactions can significantly alter the isotopic abundance of these molecules. For carbon the isotopic exchange reaction (Watson, Anicich, and Huntress 1976),

$$
{ }^{13} \mathrm{C}^{+}+{ }^{12} \mathrm{CO} \leftrightarrow{ }^{13} \mathrm{CO}+{ }^{12} \mathrm{C}^{+}+\Delta \mathrm{E}(=35 \mathrm{~K}),
$$

can enhance the ${ }^{13} \mathrm{CO}$ abundance, particularly at low temperatures, but only if this reaction is an important pathway for producing $\mathrm{CO}$. In dense shielded regions almost all available carbon is in $\mathrm{CO}$ and the $\mathrm{CO}$ isotope ratio reflects the elemental isotopic ratio, ${ }^{12} \mathrm{CO} /{ }^{13} \mathrm{CO} \cong$ ${ }^{12} \mathrm{C}_{\text {true }}{ }^{13} \mathrm{C}_{\text {true. }}$. This result does not necessarily hold true for the other carbon molecules. The destruction rate of ${ }^{13} \mathrm{C}^{+}$is larger than that for ${ }^{12} \mathrm{C}^{+}$and therefore less thirteen carbon (in the form of ions and neutrals) is available to form other molecules through the nonisotope exchange reactions (cf. detailed discussion in Langer et al. 1984). Therefore, $\mathrm{H}_{2}{ }^{12} \mathrm{CO} / \mathrm{H}_{2}{ }^{13} \mathrm{CO}, \mathrm{H}^{13} \mathrm{CN} / \mathrm{H}^{13} \mathrm{CN}$, etc. $<{ }^{12} \mathrm{C}_{\text {true }} /{ }^{13} \mathrm{C}_{\text {true. }}$. Model calculations (Langer and Graedel 1989) show that formaldehyde can be enhanced in the twelve carbon species by up to a factor of two even when CO is not (or only slightly) enhanced with respect to thirteen carbon species. This aspect of the isotopic chemistry may explain the difference in the isotopic ratios derived from $\mathrm{CO}$ and formaldehyde in the Galactic surveys, but more observations are needed to confirm this explanation.

In UV dominated regions (cloud edges, PDRs) there is sufficient UV to keep some carbon ionized and therefore the isotopic exchange reaction can be very important for $\mathrm{CO}$. In addition, $\mathrm{CO}$ is photodissociated by line absorption and self-shielded to different degrees depending on their relative abundances (cf. Bally and Langer 1981; van Dishoeck and Black 1988). Under such conditions the less abundant species may be isotopically underabundant relative to the more abundant species and the observed isotope ratios will not reflect the true elemental isotopic ratio. This regime of the interstellar chemistry has been extensively modeled by van Dishoeck and Black (1988). Their models confirm that combinations of isotopic exchange and self-shielding lead to varying degrees of enhancement in both ${ }^{12} \mathrm{CO}$ and ${ }^{13} \mathrm{CO}$.

In the UV regions this variability of $\mathrm{CO}$ enhancement can also impact the isotopic abundances of the other carbon molecules. Any isotopic enhancement of $\mathrm{CO}$ (no matter 
which isotope) results in the reverse enhancement in the exchange ion $\mathrm{C}^{+}$and hence its direct products, such as $\mathrm{CH}$ and $\mathrm{CH}_{2}$. In addition, for $\mathrm{CN}$ there is the possible exchange reaction,

$$
{ }^{13} \mathrm{C}^{+}+{ }^{12} \mathrm{CN} \leftrightarrow{ }^{13} \mathrm{CN}+{ }^{12} \mathrm{C}^{+}+\Delta \mathrm{E}(=34 \mathrm{~K}),
$$

which will be important when it competes with photdestruction of CN. A simple model of $\mathrm{CN}$ formation from reactions of $\mathrm{N}$ with $\mathrm{CH}, \mathrm{CH}_{2}, \mathrm{C}_{2}$, and $\mathrm{C}_{2} \mathrm{H}$ (whose sum is denoted by $\mathrm{CM}$ ) leads to the following relationship for the carbon isotopic ratio of $\mathrm{CN}$,

$$
\left[{ }^{12} \mathrm{CN} /{ }^{13} \mathrm{CN}\right] \approx\left[{ }^{12} \mathrm{CM} /{ }^{13} \mathrm{CM}\right] \frac{\left[\Gamma(\mathrm{CN})+\mathrm{k}_{f} \mathrm{n}\left({ }^{12} \mathrm{C}^{+}\right)+\mathrm{k}_{0} \mathrm{n}(\mathrm{O})\right]}{\left[\Gamma(\mathrm{CN})+\mathrm{k}_{\mathrm{r}} \mathrm{n}\left({ }^{12} \mathrm{C}^{+}\right)+\mathrm{k}_{0} \mathrm{n}(\mathrm{O})\right]}
$$

where, $\Gamma$, is the photodestruction rate, $\mathrm{k}_{\mathrm{f}}$ and $\mathrm{k}_{\mathrm{r}}$ are the forward and reverse isotope exchange reaction rate coefficients, $\mathrm{k}_{0}$ the neutral-neutral reaction rate coefficient with oxygen, and $n(X)$ the density of species $X$. From this expression it can be seen that the $\mathrm{CN}$ isotope ratio can be enhanced either in twelve or thirteen $\mathrm{C}$ depending on conditions. The isotope exchange chemistry for $\mathrm{CN}$ may explain the variability in the $\mathrm{CN}$ observations and the differences between the $\mathrm{CN}$ and $\mathrm{CH}^{+}$isotope ratio in the same sources. $\mathrm{CH}^{+}$is not expected to be isotopically enhanced since it is thought to be formed under high temperature conditions where the difference in isotopic rates is unimportant.

\section{References}

Bally, J. and Langer, W. D. 1981, Ap. J., 255, 143.

Crane, P. and Hegyi, D. J. 1988, Ap. J. (Letters), 326, L35.

Crane, P., Hegyi, D. J., and Lambert, D. L. 1991, Ap. J., 378, 181.

van Dishoeck, E. and Black, J. 1988, Ap. J., 334, 711.

Hawkins, I., Jura, M., and Meyer, D. M. 1985, Ap. J. (Letters)., 294, L131.

Hawkins, I. and Jura, M. 1987, Ap. J., 317, 926.

Henkel , C., Wilson, T. L., and Beiging, J. 1982, Astr. Ap., 82, 41.

Henkel , C., Walmsley, M., and Wilson, T. L. 1980, Astr. Ap., 109, 344.

Kaiser, M. E., Hawkins, I., and Wright, E. 1991, Ap. J., in press.

Langer, W. D., Graedel, T. E., Frerking, M. A., and Armentrout, P. B., 1984, Ap. J., 277,581 .

Langer, W. D.and Graedel, T. E., 1989, Ap. J. Suppl., 69, 241.

Langer, W. D. and Penzias, A. A. 1990, Ap. J., 357, 477 (LP90).

Langer, W. D. and Penzias, A. A. 1991, submitted to Ap. J., (LP91).

Prasad, S. S. and Huntress, W. T., Jr. 1980, Ap. J. Suppl., 43, 103.

Stahl, O., Wilson, T. L., Henkel, C., and Appenzeller, I. 1989, Astr. Ap., 221, 321.

Stahl, O. and Wilson, T. L. 1991, Astr. Ap. (Letters), in press.

Vladilo, G. and Centurion, M. 1991, Astr. Ap., in press. 